(c) (i) (⿹)

\title{
La ramificación ontológica: evaluación crítica de la antropología contemporánea*
}

JUAN CAMILO PERDOMO-MARÍN ${ }^{* *}$

Artículo de revisión sobre los debates de la antropología contemporánea.

Recibido: 27 de junio de 2019 - Evaluado: 3 diciembre del 2019 - Aceptado: 3 de febrero del 2020

Citar como: Perdomo-Marín, J. C. (2020). La ramificación ontológica: evaluación crítica de la antropología contemporánea. Hallazgos, 17(34), 273-302. Dor: https://doi.org/10.15332/2422409X.5283

El artículo deriva de un ejercicio investigativo realizado de manera independiente.

** Antropólogo y docente catedrático de la Universidad de Caldas, Colombia.

Correo electrónico: juancaperdo@hotmail.com

ORCID: 0000-0003-2714-455X 


\section{Resumen}

Este artículo presenta una revisión de los debates ontológicos en la antropología contemporánea. Para esta tarea, primero, se identificarán las condiciones históricas y epistemológicas que posibilitaron y guiaron su surgimiento; segundo, se caracterizarán panorámicamente sus ramificaciones teóricas, haciendo hincapié en los elementos comunes que las agrupan, junto con las particularidades y los desencuentros que las diferencian; por último, se realizará una evaluación crítica de sus principales ponentes, con el fin de develar posibles falencias y potencialidades. Este ejercicio, en suma, permitirá comprender lo que puede significar hacer antropología en el presente y hacia a dónde tiende a apuntar su futuro.

Palabras clave: antropología, debate cultura/naturaleza, epistemología, ontología, representación. 


\title{
Ontological ramification: critical evaluation of contemporary anthropology
}

\begin{abstract}
This article presents a review of the ontological debates in contemporary anthropology. For this task, first, the historical and epistemological conditions that enabled and guided its rise will be identified; second, its theoretical ramifications will be characterized in an all-encompassing manner, emphasizing the common elements that bring them together, along with the particularities and disagreements that differentiate them; finally, a critical evaluation of its main exponents will be made, in order to uncover possible shortcomings and potentialities. This exercise, in short, will allow to understand what it can mean to do anthropology in the present and where its future tends to point.
\end{abstract}

Keywords: anthropology, culture/nature debate, epistemology, ontology, representation.

\section{Ramificação ontológica: avaliação crítica da antropologia contemporânea}

\section{Resumo}

Este artigo apresenta uma revisão dos debates ontológicos na antropologia contemporânea. Para isso, são identificadas as condições históricas e epistemológicas que possibilitaram e guiaram seu surgimento; em seguida, são caracterizadas panoramicamente suas ramificações teóricas, com ênfase nos elementos comuns que as agrupam, junto com as particularidades e os desencontros que as diferenciam; por último, é realizada uma avaliação crítica de seus principais representantes, a fim de revelar possíveis falhas e potencialidades. Esse exercício permitirá compreender o que pode significar fazer antropologia no presente e para onde se dirige seu futuro.

Palavras-chave: antropologia, debate cultura/natureza, epistemologia, ontologia, representação. 
¿Cómo se puede distinguir el hombre de ciencia moderno de un mago o de un adivino o, incluso más allá de las sociedades humanas, de la bacteria que interroga también al mundo y que no cesa de poner a prueba el reconocimiento de las señales químicas en función de las cuales se orienta?

PRIGOGINE Y STENGERS La nueva alianza: metamorfosis de la ciencia (2004, p. 31)

\section{Introducción}

La antropología ha sido y es una disciplina tan heterogénea en términos temporales y espaciales que no es posible definirla a partir de una lista exacta de principios plenamente compartidos que la dotarían de una identidad propia. De hecho, si se compara algún texto de uno de sus fundadores como Edward Tylor con una investigación actual, por medio de sus lenguajes, estrategias y propósitos, se podría creer que se están leyendo dos disciplinas diferentes. Debido a su amplia multiplicidad de voces es necesario explorar una comprensión que parta, no de la pregunta metafísica del qué es, sino de una interrogación pragmática por el cómo la antropología ha construido sus objetos de estudio.

Este segundo camino implica considerar que la antropología, como toda disciplina, es un escenario de problematización. Esto se debe a que ha elaborado múltiples objetos de investigación al integrar de forma contingente preguntas, epistemologías, metodologías, intereses políticos y debates de otras disciplinas. Aun así, esta identidad relacional no vuelve totalmente difusa su especificidad académica, puesto que su interés, creatividad y experimentación pretende resolver, desde ángulos sumamente dispares, interrogantes parcialmente comunes.

Por un lado, los enfoques antropológicos buscan comprender las lógicas y las fuerzas que guían el-comportamiento-y-la-significación de los seres humanos. Este eje ha sido abordado desde diferentes perspectivas. Dentro de las más centrales se encuentran las preguntas biologicistas, culturalistas y bio-culturales que indagan los límites entre la naturaleza y la cultura. Por el otro, dichos enfoques han pretendido elaborar herramientas descriptivas para representar las diversas prácticas-y-conceptualizaciones de los grupos humanos. De este modo se han centrado en la construcción de estrategias etnocéntricas, poéticas y colaborativas para responder a la pregunta: ¿cómo dar cuenta de la experiencia-de-mundo de los diversos grupos humanos? 
Vale la pena señalar que la redefinición de los límites entre la naturaleza y la cultura no ha sido una tarea exclusiva de la antropología. La fenomenología, el psicoanálisis, el feminismo, entre otros, también han confrontado los esencialismos biologicistas; y, a su vez, la genética, la historia ambiental y las ciencias cognitivas, los esencialismos culturalistas. Aun así, esta disciplina posee una oportunidad especial para abordar dichos límites, puesto que sus debates en torno a la representación etnográfica le han obligado a preguntarse críticamente si la dicotomía cultura/naturaleza es un marco neutro o no de descripción de la interacción humana con el mundo.

En la comparación transcultural las dos preguntas centrales de la antropología son entrelazadas a razón de que comprender las acciones del Otro conlleva interrogar por el cómo es posible figurarlas, y, a su vez, la representación del Otro implica un entendimiento particular de la realidad para el investigador. Por consiguiente, las dificultades que surgen en campo al momento de dar cuenta del mundo social del Otro lo conducen a relativizar y problematizar sus propios marcos ontológicos, puesto que sus premisas parten de una representación específica de la realidad —es decir, un conjunto básico de metáforas para concebir las relaciones, entidades y contextos existentes - que pueden limitar su sensibilidad etnográfica. De este modo, las preguntas en torno al análisis de los límites entre la naturaleza y la cultura, y por el cómo dar cuenta de la experiencia-de-mundo del Otro, han confluido directamente en la problematización ontológica y representacional por parte del investigador, y, en consecuencia, le han dado el sello disciplinar a la antropología como la familiarización de lo extraño y la extrañeza de lo familiar.

A partir de la década de los ochenta se comenzó a perfilar una nueva identidad antropológica que problematiza la extrapolación, la comprensión y la existencia misma de lo que entendemos por sociedad, humanidad, agencia, intencionalidad, etc. Si bien estos debates son herederos directos de las dos preguntas antropológicas anteriores, las amplifican en gran medida, a razón de que no pretenden identificar fronteras causales entre la naturaleza y la cultura, sino que procuran desafiar las bases de la metafísica occidental al implosionarlas. Y, paralelamente, buscan replantear la idea de representación al tomar en cuenta en el proceso de contextualización a las entidades no-humanas como parte de la constitución y el mantenimiento del mundo social. De este modo el ser humano empieza a ser paulatinamente descentrado como foco de análisis y conceptualización.

El presente artículo realizará un recorrido genealógico, comparativo y evaluativo para estudiar los diferentes debates ontológicos actuales de la antropología. En el primer apartado se señalarán las condiciones históricas y epistemológicas 
que posibilitaron y guiaron su surgimiento. En el segundo, se caracterizarán panorámicamente sus ramificaciones académicas, haciendo hincapié en los elementos comunes que las agrupan, junto con las particularidades y los desencuentros que las diferencian. Por último, se realizará una evaluación crítica de sus principales ponentes con el fin de develar sus posibles debilidades y potencialidades.

\section{La antropología como crítica al universalismo}

Antes de desechar apresuradamente los debates ontológicos de la antropología contemporánea, al satanizarlos como un nuevo posmodernismo relativista y una nueva moda académica vacía, o, de forma inversa, celebrarlos como una ruptura radical ante el legado cartesiano de las ciencias sociales que puede descolonizar la disciplina, es necesario evaluar la legitimidad de la problematización a la que responden sus teorizaciones.

A grandes rasgos, desde una postura realista los diferentes debates antropológicos clásicos (desde el evolucionismo y pasando por el funcionalismo, exceptuando el particularismo histórico) biologizaron a la cultura, la consideraron como una respuesta práctica a los condicionamientos y las potencialidades objetivas de la naturaleza. En esa medida, el ejercicio del antropólogo consistía en representar el mundo cultural para mostrar los principios adaptativos que lo guiaban. En oposición, los debates modernos (desde el estructuralismo en adelante) irrumpieron en el panorama académico al centrase en estudiar la relación entre el pensamiento, el lenguaje y la cultura, por lo que, junto con los debates posmodernos (enmarcados en la crisis de la representación en los setenta y ochenta), resaltaron el papel arbitrario de los mundos simbólicos en relación con las condiciones ambientales del mundo. Por consiguiente, desde estas lecturas la cultura sería una propiedad autónoma ante la naturaleza. Por ello Sahlins ([1976]1988) sostenía:

Nunca hay un verdadero diálogo entre el silencio y el discurso: por un lado, las leyes naturales y las fuerzas "independientes de la voluntad humana” y, por el otro, el sentido que tal o cual grupo de hombres se asigna a sí mismos y asigna a su mundo. (1998, p. 61)

Esta apuesta culturalista tenía a su vez una implicación crítica frente a los debates anteriores de la representación, debido a que pretendía desnaturalizar los órdenes sociales de los énfasis biologicistas, evidenciando la arbitrariedad cultural de, 
por ejemplo, el género, la memoria, la alimentación, el poder, el dolor, etc. Si bien el horizonte intelectual de la antropología parecía haber sido clausurado por los estudios culturalistas, dichos debates entrarían en crisis desde finales de los años setenta. Esto sucedió a causa de que la idea de cultura como una totalidad conllevaría amplias dificultades metodológicas y teóricas, debido a que poseía un límite lógico, conducía a la tendencia generalizante de considerar a priori que todo puede ser explicado como una "construcción social”.

Frente al último punto, dicho argumento constructivista encerró a la antropología en un callejón sin capacidad explicativa, puesto que daba por sentado lo que pretendía explicar. Así, por ejemplo, el posestructuralismo, como heredero de estos debates, afirmaría que la realidad es construida discursivamente pero que no todo es discurso. Aun así, sería incapaz de decir algo significativo sobre la realidad extradiscursiva y no-humana, debido a su enclaustramiento en una comprensión antropocéntrica de la realidad que reduce las relaciones sociales a vínculos inmateriales y la materialidad del mundo al poder humano. Y es de resaltar que estos últimos temas se volvieron cada vez más urgentes de resolver debido a los crecientes debates en torno al cambio climático y al mundo tecnológico, los cuales reposicionaron paulatinamente a la naturaleza como centro de análisis en las ciencias sociales (Milton, 1996; Latour 2007, 2008; Tsing, 2015).

Es a finales de los años ochenta cuando surge la antropología contemporánea como respuesta crítica y operativa a las falencias del biologicismo y el culturalismo y la crisis de la objetividad científica (debido a los debates de la filosofía de la ciencia, el feminismo y el poscolonialismo). Estos factores encausaron y obligaron a que la antropología confrontara la rigurosidad de sus propias categorías de análisis, ejercicio que implicó la elaboración de nuevas estrategias para contextualizar el objeto de estudio, lo que, en consecuencia, lo trasformó.

La problematización de la antropología ante el estatus ontológico de sus categorías de estudio fue un ejercicio de identificación y confrontación interna de sus propias generalizaciones universalistas. El motivo central fue que se reconoció que, por ejemplo, los términos como estructura, sistema y sociedad no operaban como medios descriptivos sino prescriptivos, es decir, predelimitaban los datos que se iban estudiar y ocultaban sus cargas políticas. Es por este motivo por el que surgieron las primeras críticas a la extrapolación del dualismo cultura/naturaleza en sociedades no modernas (Strathern 1980; Povinelli, 1995; Arhem, [1996]2001; Descola, [1996]2001), al concepto mismo de cultura (Wagner, [1975]1981; Abu-Lughod, [1991]2012; Gupta y Ferguson, [1992]2008), de naturaleza (Ingold, [1991]2013a, 1993) y de sociedad (Wolf, 
1988; Strathern, Peel, Toren y Spencer, [1989]1996; Latour, [1991]2007). Esto condujo a un renovado interés por el mundo objetual (Appadurai, [1986]1991) y animal (Ingold, 1994) y su interrelación (Callon, 1986), reposicionando lo social más allá de los seres humanos.

A comienzos de los años noventa se dio un nuevo viraje disciplinar. Comenzó la proliferación y la complejización de los debates antropológicos que buscarían abandonar la oposición cultura/naturaleza, por un lado, dándole mayor relevancia a los estudios sobre la corporalidad y las prácticas sociales para impugnar la centralidad analítica del discurso y, por el otro, elaborando estrategias metodológicas para conceptualizar por igual a los humanos y los no-humanos. Paralelamente a esto se daría un florecimiento de investigaciones que retomarían la vocación comparativa (de la antropología moderna), al replantear la comprensión de lo local y lo global, criticando el énfasis particularista de la antropología posmoderna por medio de la yuxtaposición de diferentes escalas y procesos de análisis, y el ejercicio de reflexividad (de la antropología posmoderna), confrontando la falta de objetivación autocrítica del investigador de la antropología moderna, para evitar así falsos universalismos al utilizar creativamente la experiencia en campo como un medio de innovación teórica.

Puntualmente, las problematizaciones contemporáneas comenzaron a elaborar teorías y metodologías experimentales para responder al agotamiento explicativo del constructivismo. Por ello, como señalan Bryant, Srnicek y Harman (2011), las filosofías continentales, herederas de los debates de Kant (que postulan que no se puede acceder al mundo en sí mismo puesto que las categorías con las que contamos limitan su aprehensión) al sobreenfocarse en lo cultural, el lenguaje y el poder, carecieron de herramientas para analizar el mundo no-humano; al solo ver mediaciones discursivas cayeron en el escepticismo filosófico. Y esta renuncia a estudiar la realidad no discursiva ha sido un limitante para comprender problemáticas actuales:

Ante la inminente catástrofe ecológica, y la creciente infiltración de la tecnología en el mundo cotidiano (incluyendo nuestros propios cuerpos), no es claro que la posición anti-realista esté equipada para hacer frente a estos desarrollos. El peligro es que la variedad dominante anti-realista de la filosofía continental no solo ha alcanzado un punto de rendimiento decreciente, sino que ahora limita activamente las capacidades de la filosofía en nuestro tiempo. (2011, p. 3)

En medio de este devenir intelectual se pueden rastrear en los debates ontológicos tres textos paradigmáticos que han marcado la historia reciente de la antropología. El primero es Naturaleza y sociedad: perspectivas antropológicas, de Descola 
y Pálsson ([1996]2001), en el que estos autores realizaron una compilación fundacional que abrió los debates que problematizaron la división cultura/naturaleza. El segundo es Pensando a través de las cosas: teorizando los artefactos etnográficamente, en el que Henare, Holbraad y Wastell (2007) congregaron diferentes trabajos etnográficos que trascendieron el dualismo ontológico por medio de diversas propuestas experimentales. Y el tercero es Antropología y naturaleza, en el que Hastrup (2014) reunió los nuevos avances reflexivos en torno a la conceptualización y descripción del entrelazamiento del ser humano con el mundo no-humano.

En suma, parte del panorama histórico de la antropología contemporánea se condensa en un conjunto de preguntas que han comenzado a dictaminar su rumbo actual: ¿cómo es posible conocer la especificidad existencial de la experiencia humana sin proyectar los marcos ontológicos del investigador? ¿El mundo material puede ser considerado como mediador y partícipe de la interacción humana? ¿Las relaciones ecológicas pueden ser entendidas como relaciones sociales? Estos interrogantes emergentes, aunque no agoten las amplias discusiones de la antropología, abren nuevas posibilidades intelectuales para las antropologías-por-venir.

\section{Panorama de los debates ontológicos}

Los debates que problematizan el estatus ontológico de la naturaleza, la cultura y la sociedad, y comienzan a teorizar sobre la capacidad de agencia de entidades nohumanas, se tienden a enmarcar comúnmente en la categoría de "giro ontológico", agrupando a autores como Phillipe Descola, Marilyn Strathern, Eduardo Viveiros de Castro, Tim Ingold, Bruno Latour, Roy Wagner, Eduardo Kohn, Martin Holbraad, Anna Tsing, entre muchos otros.

Es llamativo el hecho de que la comprensión de este "giro", a pesar de sus genealogías unificadoras (Henare, Holbraad, y Wastell, 2007; Viveiros de Castro, 2015), sea marcadamente heterogénea, puesto que se encuentran múltiples versiones de qué es y a quiénes integra. Esta imprecisión se manifiesta en posturas como, por ejemplo, la de Ingold (Simonetti y Espirito, 2016) quien lo critica y no se reconoce como partícipe. En cambio, Descola (2014) sostiene que sí es integrante de dichos debates y afirma que estos comparten la consideración de

[...] que las fuentes de la pluralidad de seres y regímenes de existencia se encuentran en un nivel más profundo que el sociocultural, el cual es estudiado tradicionalmente por la antropología. Este nivel, que puede denominarse antepredicativo 
o emergente [...] es aquel en el que humanos y no-humanos se vuelven conscientes del otro y desarrollan modos de relacionarse anteriores a los procesos usuales de categorización y comunicación incrustados en marcos histórica y lingüísticamente contingentes. (2014, p. 268)

Pero para Holbraad y Pedersen (2017) ni Descola, Latour, Ingold e Kohn entrarían en este giro, sino autores que se realizan preguntas y apuestas ramificadas de los trabajos de Wagner, Strathern y Viveiros de Castro. De hecho, su definición sería diferente a la propuesta de Descola. Para ellos el giro ontológico es una apuesta metodológica de apertura sensible que amplía los “potenciales analíticos” de la antropología (la reflexividad, la conceptualización y la experimentación) para repensar la descripción etnográfica dándole primacía sobre la teorización. De este modo, para el giro ontológico "el problema epistemológico de cómo ver las cosas se convierte en la pregunta ontológica de qué hay que ver en primer lugar” (Holbraad y Pedersen, 2017, p. 6).

Es posible citar muchas otras percepciones divergentes, pero el punto para resaltar es que la categoría de giro ontológico es altamente confusa. Si bien agrupa autores con problematizaciones similares, la crítica al binarismo cultura/naturaleza y a las estrategias previas de representación en la antropología, a su vez, engloba debates claramente diferentes. Estas propuestas brindan soluciones experimentales marcadamente heterogéneas; además se realizan de forma mutua fuertes críticas epistemológicas, y amplían sus debates con otras disciplinas volviendo cada vez más porosa la identidad de la antropología. Y como consecuencia de la falta de especificidad de dicha categoría, diversos investigadores han combinado irreflexiva y forzadamente teorizaciones altamente disímiles. Por este motivo se omite que existen diferentes vertientes las cuales tienen un entendimiento particular de lo que cuenta como agencia, animismo, ontología, dualismo, mundo, sujeto, representación, nohumano, entre otros.

Ahora bien, la antropología colombiana ha resonado productivamente con los debates ontológicos contemporáneos realizando diversos aportes investigativos (Castellanos, 2019; Escobar, 2010; Jaramillo, 2020; Londoño Sulkin, 2005; Micarelli, 2018; Ruiz-Serna, 2017), y advirtiendo sobre sus posibles falencias (Ruiz-Serna y Del Cairo, 2016; Suárez-Guava, 2019). El presente artículo profundizará en el ejercicio de revisión de dichos debates para comprender, evaluar y diferenciar las reflexiones ontológicas de la antropología. 


\section{Mapa borromeo de la antropología}

Debido a la generalidad de la categoría giro ontológico, es necesario realizar una vigilancia epistemológica para identificar detalladamente los énfasis diferenciales en las apuestas teóricas de la antropología actual. A grandes rasgos, es posible diferenciar tres grandes ramificaciones parcialmente entrecruzadas: alteridad, materialidad y habitar.

- Alteridad: este tipo de estudios se caracterizan por problematizar las pretensiones universalistas y antropocéntricas de la epistemología occidental. Para realizar esta tarea resaltan la especificidad ontológica de las categorías de análisis de los investigadores por medio de su comparación con la lógica interna de las epistemologías no modernas. De este modo, dichos trabajos confrontan principalmente las estrategias de la representación, debido a que apuestan por "tomar seriamente" la alteridad. En otras palabras, pretenden comprender las cosmovisiones en sus propios términos, puesto que estas no serían simples interpretaciones parciales de una realidad común — que solo conocería Occidente-, sino mundos con sus propias especificidades ontológicas. En suma, los enfoques de la alteridad evidencian las amplias falencias que surgen al momento de extrapolar los marcos comprensivos occidentales a contextos en los que seres no-humanos son considerados entidades con agencia en el mundo social, y de este modo permiten dimensionar la inconmensurabilidad parcial entre mundos de significado. Este reconocimiento se considera desde la etnología como un medio transversal para impugnar y crear nuevas teorías sobre el animismo y las conversiones religiosas (como autores introductorios véase: Blaser, 2009; Descola, 2012; De la Cadena, 2010; Escobar, 2010; Holbraad, 2009; Viveiros de Castro, 2010).

- Materialidad: estas investigaciones se centran en impugnar la separación entre mundo social y mundo material, mostrando que las acciones, la significación y el conocimiento emergen por medio de la interacción práctica con el mundo artefactual que rodea al sujeto, hasta el punto en el que se desdibujan los límites entre ambos. Por consiguiente, los seres humanos compondrían la realidad por medio de su articulación con agentes no-humanos. Estos estudios, vale la pena señalar, no se centran en grupos no modernos, sino que a abordan principalmente a Occidente, problematizando la producción de conocimiento en relación con la tecnología (como autores introductorios véase: Callon, 1986; Latour, 2008; Law, 2004; Mol, 2002).

- Habitar: estos trabajos critican el antropocentrismo mostrando desde un énfasis posfenomenológico que las fuerzas semiótico-materiales de seres no-humanos se entrelazan con las actividades prácticas de los humanos, edificando y 
modificando los paisajes. Por consiguiente, el medio ambiente existiría como un mundo multiespecies en el que se sobreponen múltiples realidades orgánicas en tensión y articulación. Los estudios del habitar no tienen un contexto moderno o no moderno, puesto que se interesan en analizar de forma general las ecologías compartidas entre animales, plantas, hongos, insectos, microbios (por medio de etnografías multiespecies), y elementos inorgánicos como ríos, nubes, océanos, etc. Es por ello por lo que dichas investigaciones le dan centralidad a los procesos de interacción entre humanos y no-humanos, retomando los debates de la significación en la biosemiótica y la co-evolución de la teoría de desarrollo de sistemas (como autores introductorios véase: Hastrup, 2013, Helmreich, 2009, Ingold, 2000, 2013a; Kirksey \& Helmreich, 2010; Tsing, 2015).

Oponiéndose al constructivismo, los tres énfasis mencionados no consideran que el ser humano le da significación a una realidad carente de sentido, puesto que afirman que el mundo en sí mismo sería significativo debido a que sería un escenario de participación de actores no-solo-humanos. Esto se debe a que, a grandes rasgos, el enfoque en la alteridad ve mundos experienciales parcialmente inconmensurables; la materialidad, mundos prácticos en construcción a partir de la interrelación con no-humanos (principalmente artefactos), y el habitar, mundos orgánicos en crecimiento en el que el ambiente y el organismo-persona poseen una relación de co-constitución.

\section{Principales ponentes}

En este apartado se introducirán los autores más reconocidos de cada ramificación contemporánea para que los lectores tengan una indicación general de sus especificidades.

- Alteridad: Eduardo Viveiros de Castro (2004, 2010, 2015), por medio de su trabajo de campo y diversas etnografías en la Amazonia brasileña, establece que en las cosmologías amerindias existe un principio perspectivista en el que los no-humanos se ven a sí mismos como humanos, y a los humanos como no-humanos, mediante una lógica jerárquica de presa y depredador. De este modo, la realidad estaría constituida por puntos de vista posibilitados por los hábitos corporales de cada ser. Dichas ideas se encuentran enmarcadas en una inversión lógica, puesto que en el pensamiento amazónico no existiría una naturaleza singular en contraste con múltiples culturas, que poseerían interpretaciones parciales sobre esta 
(concepción moderna multiculturalita), sino múltiples naturalezas (concepción multinaturalista) con una sola cultura o agencia compartida por diferentes seres no-humanos.

Phillipe Descola $(2001,2012)$, con base en una vasta revisión de material etnográfico de todo el planeta, en conjunto con su trabajo en la Amazonia peruana, propone considerar que coexisten cuatro grandes ontologías (naturalismo, animismo, analogismo y totemismo), que se clasifican según las relaciones de discontinuidad y continuidad entre lo que denomina fisicalidad (cuerpo, materia, sustancias) e interioridad (temperamento, intención, emociones). Su ejercicio comparativo se basa en un modelo estructural en el que las ontologías se ordenan en principios combinatorios básicos que permiten sintetizar y dar coherencia a la variabilidad humana. Si bien estas ontologías se mezclan parcialmente, se tienden a sistematizar en una, la cual delimita y guía la lógica y la experiencia particular del sujeto ante su mundo.

- Materialidad: Bruno Latour $(2007,2008)$, de acuerdo con sus trabajos en los estudios sociales de ciencia y tecnología, sustenta críticamente que la modernidad es la promesa siempre fallida de separación entre la naturaleza y la cultura, la cual no haría más que crear híbridos naturaleza-cultura que oculta. A partir de la teoría del actor-red, afirma que la acción social no se limita a la simple interacción humana, lo que implicaría un mundo sin objetos. Por el contrario, esta acción es una conexión permanente entre humanos y no-humanos que debemos ver de forma simétrica para reconocer que estos últimos poseen capacidad de agencia. Por este motivo, su trabajo es la apuesta de rastrear las relaciones materiales que componen y mantienen el mundo.

- Habitar: Tim Ingold (1993, 2000, 2011, 2013a) estudia las relaciones ecológicas de los humanos con su ambiente. Ello lo conduce a un enfoque que critica el culturalismo de las ciencias sociales y las teorías neo-darwinianas de la biología. Su propuesta señala que los elementos del mundo no esperan para tener un significado a partir de las representaciones mentales (como en Durkheim y Lévi-Strauss), sino que mediante relacionamiento experiencial con un mundo en el que estamos inmersos por medio de flujos materiales surge la capacidad de significación; por consiguiente, esta no se encontraría preconfigurada en medios simbólicos, sino que emergería por medio de prácticas de atención. De este modo, sus reflexiones enmarcan una crítica directa a los modelos representacionales del conocimiento para centrarse en los procesos de exploración vital de los organismos-persona con el ambiente. 


\section{Evaluación de los debates ontológicos}

Es necesario resaltar que las propuestas caracterizadas no irrumpen en el panorama académico de la antropología, sino que utilizan materiales analíticos antiguos de esta disciplina y los amplían ${ }^{1}$. Debido a que cada teórico retoma diferentes legados intelectuales, no es posible unificar sus propuestas bajo la rúbrica común de giro ontológico; por ello mismo es que entre estos autores se formulan fuertes críticas.

\section{Crítica a la alteridad}

Para Tsing (2015) hay una diferencia puntual entre las ontologías alternativas y haciendo-mundos, que en este escrito son llamadas respectivamente abordajes de la alteridad y enfoques de la materialidady del habitar:

Los proyectos Haciendo-mundo, como las ontologías alternativas, muestran que otros mundos son posibles. Haciendo-mundo, sin embargo, nos enfoca en las actividades prácticas en lugar de las cosmologías [...] Aunque la mayoría de los académicos usan la ontología para segregar perspectivas, una a la vez, pensar a través del Haciendo-mundos permite una fricción estratificada e histórica consecuente. (p. 292)

De hecho, los enfoques de la alteridad, al contrastar el naturalismo moderno y el animismo no moderno como marcos ontológicos opuestos, entre lecturas dualistas de la realidad que conciben mundo pasivo versus lecturas relacionales que habitan un mundo vivo, hacen generalizaciones que omiten la complejidad misma de Occidente. Por esto Candea (2012) afirma: "El naturalismo occidental se ha convertido en consecuencia en un 'otro' antropológico exótico y desconocido en el corazón de nuestro 'yo' imaginado” (p. 37). Por ello para Candea el naturalismo antes de ser una concepción clara y estable es más bien un "horizonte" difuso de oposición que se debe confrontar, puesto que Occidente nunca ha sido enteramente naturalista, a razón de

1 Los debates actuales entre Descola e Ingold se pueden remontar respectivamente al debate clásico de la filosofía entre racionalistas y empiristas. La estrategia tipológica de Descola, que responde a la comprensión clasificatoria de Durkheim, fue usada anteriormente por Mary Douglas. La idea de múltiples mundos de Viveiros tiene referentes tan antiguos como el perspectivismo de Nietzsche, la teoría biológica de Uexküll y la postura relativista de Edward Sapir (1963): "Los mundos en los que viven diferentes sociedades son mundos distintos, no simplemente el mismo mundo con diferentes designaciones ligadas" (p. 162). En el caso de Latour, el principio de simetría que propone entre humanos y no-humanos fue abordado en los setenta por la antropología ambiental de Roy Rappaport y el enfoque ecosistémico de Gregory Bateson. 
que la frontera entre la naturaleza y la cultura nunca ha sido delimitada en sus prácticas. En sintonía con estas ideas, Sahlins (2014), analizando críticamente a Descola, sostiene que existen múltiples naturalismos que no son tan alejados del animismo, puesto que "no debemos dejar que la presunción del naturalismo oscurezca la facilidad con que y a menudo dotamos a los 'cuerpos' sociales [...], así como a algunos animales y cosas inorgánicas, con rasgos de personalidad humana” (p. 288)².

Por su parte, Graeber (2015) afirma que este tipo de estudios cae en el exotismo, en el idealismo filosófico, en la exageración, en la sistematicidad y en la coherencia de las creencias de las personas, y partiría de un moralismo esencialista que busca una nueva metafísica. Debido a lo anterior, se pregunta si los teóricos del "giro ontológico”: “¿realmente creen que la mayoría de las personas que los antropólogos estudian realmente estarían de acuerdo con la proposición de que viven en una 'naturaleza' u 'ontología' fundamentalmente diferente a la de otros humanos [...]?" (p. 34). Las reflexiones críticas de Hugh-Jones (2019) sobre los estudios de Descola y Viveiros sobre la relación humano-animal en la Amazonía parecieran confirmar la denuncia de Graeber. Las teorías generalizantes de estos antropólogos omitirían la heterogeneidad, la contradicción y complejidad de los datos de campo. De allí que crearían falsas fronteras entre los amerindios y los euro-norteamericanos, por lo que "estos contrastes representan las puntas ontológicas bien delineadas de icebergs etnográficos grandes y deformes” (p. 163).

\section{Viveiros de Castro: generalizaciones del perspectivismo}

Es posible encontrar críticas que indican como el énfasis excesivo en el estudio de las cosmovisiones conduce a omitir las dimensiones políticas y materiales de los grupos humanos. Así, por un lado, en relación con las representaciones identitarias, Ramos (2012) señala que el perspectivismo "es indiferente a las consideraciones políticas en cuanto a los predicamentos de las personas indígenas en contextos interétnicos

2 Ingold (2000) agrega: "Incluso los habitantes de países nominalmente occidentales ninguno de ellos resulta ser un occidental de sangre pura, o incluso ser particularmente moderno en su enfoque de la vida; y que la tradición occidental de pensamiento, examinada de cerca, es tan variada, multívoca, históricamente cambiante y disputada como cualquier otra" (pp. 6-7). De forma similar, Strathern (1980) considera que "no se puede dar un significado único a la naturaleza o la cultura en el pensamiento occidental; no hay una dicotomía consistente, solo una matriz de contrastes" (p. 177). 
adversos" (p. 438) ${ }^{3}$. En otras palabras, este ignora que diversas relaciones de poder median en las expresiones identitarias de las comunidades indígenas. De hecho, se ha denunciado que en los eventos académicos los "representantes indígenas parecen sentirse ventriloquizados por el discurso del perspectivismo Amerindio” (Swanson, Bubandt y Tsing, 2015, p. 157).

Por el otro lado, el perspectivismo de Viveiros de Castro le da, según SantosGranero (2012), un lugar secundario a los objetos en el sostén de las cosmologías amerindias y en la construcción de los cuerpos, en contraste con el énfasis excesivo hacia los animales. En relación con este punto, según Hornborg (2015), al no analizar las relaciones de fetichización entre sujetos-artefactos, el perspectivismo se encuentra "confinado a las representaciones humanas de perspectivas no-humanas. Nunca será capaz de decir nada específico acerca de cómo no-humanos experimentan actualmente al mundo" (p. 38).

Sumado a lo anterior, diversas investigaciones etnológicas critican la generalización de sus postulados en las diferentes comunidades de la Amazonía, principalmente el sobreénfasis en su modelo predador. Así, por ejemplo, Brabec de Mori (2013) afirma que en la ontología de los shipibo los límites entre humanos y no-humanos:

[...] no se basan en una jerarquía de depredadores y presas, como comúnmente sugiere el perspectivismo, ni replican una economía simbólica de afinidad. La jerarquía se basa en las competencias de percepción y acción de los agentes, y la escala es muy ajustada y flexible. (p. 356)

\section{Phillipe Descola: riesgos de la tipología}

La tipología postulada por Descola, según Sahlins (2014), proyecta una mayor diferencialidad entre concepciones de mundo similares, puesto que opone radicalmente lógicas basadas en los mismos principios antropomórficos ${ }^{4}$. De hecho, Hanks y Severi (2014) exponen que no existen ontologías delimitadas y sistemáticas como propone el modelo de Descola, puesto que en un mismo contexto se desarrollan múltiples procesos cognitivos de traducción.

3 Por su parte, Ingold (2016a) afirma que los postulados de Descola son conservadores porque su teoría "nos trae de vuelta a las tradiciones venerables de la antropología comparativa, seguras en la academia e inafectada por la agitación intelectual y política del mundo contemporáneo” (p. 318).

4 Según Scott (2016) y Arjem (2001), las creencias animistas de los Aroshi y los Makuna, respectivamente, comparten cualidades que Descola ha señalado en el totemismo australiano. 
Estas tipologías tienen también como falencia el ser ahistóricas y homogeneizantes, lo cual lleva a considerar las diferencias ontológicas como cualidades dadas (Hornborg, 2015), como en el caso del perspectivismo (Weismantel, 2015). Por este tipo de falencias es que Vilaça (2015) expone que dicho modelo tipológico no permite entender las transformaciones ontológicas contemporáneas relacionadas con la cristianización del animismo. Estos últimos argumentos indirectamente ratifican la crítica de Ingold (2016a, 2016b), la cual sostiene que para Descola las opciones de los sujetos serían anteriores a su experiencia en el mundo, puesto que estos se encontrarían precondicionados por sus marcos ontológicos, lo cual erróneamente omitiría que estos esquemas no son pre-dados porque de hecho nunca serían alcanzados, sino que están siempre en desarrollo.

Si bien Descola no cae en un pluralismo ontológico, sino que analiza principios finitos de cuatro grandes ontologías, Ingold (2016a) señala que su universalismo relativo, en vez de ser un punto de comparación neutro, "es en verdad una versión poco disfrazada que de otra manera va bajo la rúbrica de naturalismo" (p. 303). Esto se debe a que parte de la lógica naturalista, junto con Lévi Strauss, la cual sostiene que hay un conjunto de representaciones sociales que toman forma a partir de estructuras mentales básicas, lo cual separa de forma dualista la mente de la realidad.

\section{Crítica a la materialidad}

Una crítica común que comparten los Estudios sociales en ciencia y tecnología es que aglutinan de forma generalizante e imprecisa a los artefactos y a los seres orgánicos con cualidades semióticas dentro de la categoría de no-humanos, por lo que "el resultado es una forma de dualismo en el que los humanos y los no-humanos adquieren mezclas de propiedades como-cosas y como-humanos” (Kohn, 2013, p. 92).

\section{Bruno Latour-redes difusas}

Las críticas hacia Latour se centran en su consideración sobre las redes. Strathern (1996) reprocha que la teoría del actor-red forma cadenas de cadenas como estrategia metodológica que no tiene un punto de parada, por lo que: "el poder de dichas redes analíticas también es su problema: teóricamente, no tienen límite” (1996, p. 523). Además, según Ingold (2011), Latour ve el mundo como "un ensamble de partes o piezas heterogéneas" (p. 64) que lo limitaría a ver relaciones mecánicas en una realidad que de hecho es dinámica.

Si bien Latour pretende abandonar la idea de contexto para seguir a los actores humanos y no humanos, Tsing (2010) sostiene que esto reduce "la red a los 
ensamblajes sociales más cercanos a sus informantes" (p. 50). Por eso, omitir el estudio de las partes y el todo en sus análisis, en otras palabras, no querer delimitar un contexto y cruzarlo con otros, genera, según Tsing, puntos ciegos en su teoría, puesto que le impide establecer un marco comparativo para poder figurar y darles sentido a los datos. Resonando con las críticas anteriores, Rochelau (2011) afirma que es problemático que comúnmente en la teoría del actor-red el ser humano se represente como el actor central de estas redes y, a su vez, que se parta erróneamente de la idea de "que todas las conexiones son positivas y pueden tratarse como activas" (p. 213).

De hecho, la centralidad que posee la operación de rastreo de las entidades nohumanas conduce a una falta de reflexividad por parte del investigador, a razón de que, como expone Morita (2013), "su fuerte énfasis en seguir a los actores parece relegar el problema del propio dispositivo analítico del investigador a un punto ciego" (p. 17). Es por esto por lo que la invitación de la teoría del actor-red "para minimizar la agencia del investigador a menudo oscurece la tensión entre las conexiones hechas por los actores y la propia contextualización del investigador” (Tsing, 2010, p. 5).

Otra falencia que posee la teoría de Latour es que, según Kohn (2013), para esta la intencionalidad humana se convierte en un marco oculto para dotar de agencia a los objetos, lo cual reproduce el dualismo cartesiano que se busca criticar ${ }^{5}$. Aunque si bien Latour busca crear un principio de simetría que diluya la frontera y la jerarquía entre humanos y no-humanos, para Ingold (2013b) no lo logra conseguir, a causa de que en su reflexión sobre los animales reproduce la excepcionalidad humana. Este comentario parte de la consideración de que para Latour las relaciones sociales de los animales son sumamente inestables a falta de la fijación material, en contraste con los humanos por el uso de artefactos. Pero Ingold contraargumenta esta posición sosteniendo que los animales sí poseen una interacción activa con el mundo material que les da soporte a sus relaciones sociales de interacción. Sumado a lo anterior, se ha denunciado que hay una falta de pensamiento crítico por parte de Latour ante relaciones inequitativas del poder en el marco del capitalismo (Hornborg, 2017), argumento que se puede extrapolar a Descola, Viveiros e Ingold.

5 Por esta razón, Kohn (2013) critica el hecho de que los estudios de Latour, Law, Mol y Haraway erróneamente parten de una teorización sobre los no-humanos con base en relaciones centradas en el lenguaje humano. Por esto invita a realizar una "antropología más allá de lo humano" que pueda "repensar la relacionalidad viéndola como semiótica, pero no siempre y no necesariamente como el lenguaje” (p. 84). 


\section{Crítica al habitar}

Se ha señalado que los giros analíticos hacia las plantas y los animales tienden a reproducir la separación colonial que exotiza al Otro bajo un uso altamente moralizador, puesto que, “al igual que lo 'primitivo' fue inventado -o instrumentalizado- por etnógrafos para poner en cuestión nuestro estilo de vida Occidental dado-por-sentado, hoy en día el animal es utilizado para cuestionar nuestra autodescripción como humanos” (Ress, 2016).

\section{Tim Ingold: contacto polémico}

Por su parte, las críticas a Ingold se elaboran en torno a su división entre interpretación y percepción. Milton (1996) confronta esta distinción porque considera imposible separar "el conocimiento que se deriva de las percepciones de las personas sobre su entorno y el que se deriva de sus interpretaciones (o de las de los demás) de este” (p. 61). Por su parte, Bird-David (1999) critica que Ingold, al igual que unas de sus principales influencias James Gibson, al pretender trascender el antropocentrismo, “le presta una atención inadecuada a la 'acción' interhumana en-el-mundo a favor de las 'acciones' a través de otras especies” (p. 75).

También se considera que su teorización es una propuesta metafísica. Keane (2018) afirma que "el anhelo de contacto directo e inmediato con el mundo tiene una historia venerable en el misticismo religioso y el romanticismo estético. Pero esto no es [...] un anhelo que debería guiar el trabajo antropológico” (p. 45). De forma similar, Descola (2016) considera que el conocimiento inmediato abordado en reflexiones de Ingold "es en su mayoría inaccesible para la investigación etnográfica” (p. 324). Además, agrega que su trabajo fenomenológico es

[...] una celebración metafísica de la vida 'en lo abierto' donde las diferencias colectivas entre los humanos y su explicación han desaparecido progresivamente a favor de una filosofía moralizante y altamente normativa del habitar cuya proximidad excesiva a los conceptos heideggerianos me parece inquietante. (p. 326)

A pesar de que Ingold resalta la importancia de la coparticipación con el mundo del que somos parte en la elaboración del conocimiento, Hicks (2010) asevera que sus reflexiones se quedan en un campo filosófico sin trabajo de campo: "Al hacerlo, reproduce precisamente la tendencia de buscar explicar el mundo manteniéndolo a una distancia suficiente, a pesar de la lógica apremiante de sus argumentos para alejarse de tales enfoques" (2010, p. 81). 
Por su parte, Scott (Venkatesan et ál., 2013) cree que Ingold, al poner en diálogo las prácticas animistas con filosofías continentales, erróneamente confunde afinidad con identidad. Si bien este es un ejercicio comparativo legítimo, el problema yace en que yuxtapone forzadamente sus lógicas resaltando relaciones de similitud que, por su seducción estética y argumentativa, omiten especificidades ontológicas y temporales que las diferencian. Por lo que el descubrimiento de paralelismos del animismo, por ejemplo, con la ontología de Heidegger, tiende a ser, a su vez, una forma de creación (Scott señala que esto se aplica también a Viveiros con su lectura de Deleuze y Guattari). El punto central por resaltar de Scott es que "nuestro no dualismo no es isomórfico con el de nuestros informantes”.

De forma general, la revisión presentada hasta el momento identifica que a la alteridad se le reprocha caer en el exotismo que idealiza al Otro no moderno; a la materialidad, el reproducir el excepcionalismo y la proyección antropomórfica, y el habitar, en generar una metafísica moralista del reencanto. De allí que no hay un consenso entre los autores del denominado "giro ontológico". Las críticas mutuas y la disparidad analítica entre estos casos evidencian la imprecisión de agruparlos dentro de una misma categoría. Inclusive dentro de cada énfasis no es posible encontrar una uniformidad argumentativa, puesto que, por ejemplo, en el habitar Ingold (2013b) critica la etnografía multiespecies propuesta por Kirksey y Helmreich (2010); y si bien Descola y Viveiros son herederos del estructuralismo, los demás investigadores de la alteridad confrontan las falencias de esta escuela antropológica.

Ahora bien, los debates ontológicos caracterizados poseen dos puntos altamente polémicos. Por un lado, si bien muchos de estos pretenden romper el dualismo cultura/naturaleza, comúnmente terminan por darle continuidad en sus modelos. A este respecto, Halbmayer (2012) señala:

Los mayores e inspiradores intentos de superar la gran división occidental entre la naturaleza y la cultura limitan su impacto al volver a inscribir la diferencia naturaleza/cultura en las nociones de partidad (parthood) [...] Los humanos mismos se convierten en dividuos de la naturaleza/cultura en la teorización actual. (p. 110)

6 Holbraad (2009), elaborando una crítica similar a la de Scott, advierte los riesgos de que se crea que el animismo comprobaría la ontología relacional de las teorías occidentales que impugnan el legado cartesiano o, de forma opuesta, que dichas teorías al ir más allá del dualismo ontológico permitan comprender realmente la complejidad del animismo. Ante el primer caso, Holbraad sostiene que se deben identificar las limitaciones de las teorías occidentales; en el segundo, considera necesario no solo reconocer sus resonancias, sino también diferencias con el animismo. 
Por el otro lado, una falencia muy visible es que las nuevas generaciones de antropólogos toman los diversos debates ontológicos como puntos de partida analítica que buscan comprobar y no como preguntas abiertas para la experimentación. En artículos científicos y congresos académicos se están dando por sentadas estas reflexiones teóricas, que tienen al "giro ontológico" como referente explicativo a priori. Este esnobismo ha comenzado a limitar la renovación del conocimiento antropológico.

\section{El laberinto ontológico}

En el panorama expuesto resulta impreciso posicionar dichos debates dentro de los espectros realistas o constructivistas, debido a que la especificidad de ambas categorías resulta ser sobrepasada por los virajes actuales. Piénsese, por ejemplo, en la obra de Latour. Su propuesta no es realista porque no parte de la premisa de que la realidad y el sujeto que conoce se encuentran separados; de hecho, su obra estudia su entrelazamiento. Pero tampoco es constructivista en un sentido ortodoxo, ya que sostiene que la realidad social no la construyen los seres humanos, sino que esta se elabora en el ejercicio práctico e inacabado de interacción con el mundo material. A raíz de esta nueva especificidad, en la antropología es necesario indagar categorías analíticas más precisas para describir sus lógicas comprensivas.

Actualmente los debates ontológicos tienden a enmarcarse en el relacionismo y la ontología orientada-a-objetos. El relacionalismo parte de la premisa de que las cosas del mundo se componen enteramente de la articulación de relaciones, y los ontólogos orientados-a-objetos, en que la realidad se sustenta en esencias autónomas que existen en sí mismas y no se agotan por su interacción con otras entidades (Golub, 2014; Scott, , 2016) 7 .

En la filosofía contemporánea heredera del realismo crítico se encuentran principalmente debates de la ontología orientada-a-objetos, y en la antropología priman los abordajes del relacionismo. Por un lado, en la alteridad, la etnología contemporánea señala que en diversos grupos humanos no modernos los individuos se constituirían y actuarían a partir de una alta conectividad con el mundo (en contraste con Occidente). De este modo, en el caso de Melanesia:

Cuando las relaciones se perciben como inmanentes en las cosas y las personas, las personas trabajan para darlas a conocer a través de las analogías que revelan. Las

7 Para indagar en la ontología-orientada-a-objetos véase Bryant, Srnicek y Harman (2011), y en el relacionismo, Ingold (2015b). 
personas y las cosas se descomponen para revelar las relaciones que las constituyen. (Strathern, [1991]2004, p. 79)

$Y$ en el caso latinoamericano, diversos estudios en sus trabajos etnográficos oponen ontologías dualistas (separación moderna cultura/naturaleza) y las relacionales (continuidad de grupos no modernos entre humanos y no-humanos) (Blaser y De la Cadena, 2009; Blaser, 2009; Escobar, 2010).

Las demás ramificaciones de la antropología no parten de la oposición de ontologías modernas versus no modernas, sino que se basan en la premisa ontológica relacionista como marco general de la realidad. Desde la materialidad, Latour (2008) afirma que los objetos existen por las relaciones que los constituyen. Por esto aconseja que "cuando se enfrenta a un objeto, atienda primero a las asociaciones de las que está hecho y después mire cómo ha renovado el repertorio de vínculos sociales" (p. 328). En el caso del habitar, Ingold (2015a) propone ver el mundo como un nudo de relaciones en crecimiento. Es por ello por lo que "toda cosa es un parlamento de líneas [...] estudiar tanto personas como cosas es estudiar las líneas de las que están compuestas” (p. 21).

Si bien los postulados del relacionismo poseen amplio potencial analítico, su amplia generalización es problemática. Scott (2016) señala, a partir de su estudio en Nueva Guinea entre los Arosi, que esta comunidad no simplemente parte de una cosmología no dualista, ya que sigue principios relacionales y esencialistas que se combinarían en lo que denomina una poli-ontología. Por ello Scott sostiene: "La idea que tenemos Melanesios relacionales o Amerindios perspectivistas siempre necesita ser probada y no presupuesta" (Golub, 2014) ${ }^{8}$.

En resonancia con las ideas anteriores, Pedersen (2013) critica lo que denomina el "fetichismo de la conectividad", en el que se cree que las prácticas animistas, estudiadas en Amazonas, Melanesia y Mongolia, solo buscan conectividad con el mundo. Esto sería erróneo, puesto que al enfocarse demasiado en la relacionalidad no toman en cuenta los procedimientos de corte de relaciones que realizarían los artefactos chamánicos para evitar los peligros de la cercanía con espíritus. En el mismo sentido, Hvenegaard (2016), en su estudio de los bugkalot en Filipinas, sostiene que para los pacientes de médicos tradicionales visitarlos en lugares distantes y afirmar que no

8 Scott (Golub, 2014) también agrega que las lecturas celebratorias de las ontologías relacionales, en contraste generalizante con el supuesto dualismo cartesiano y kantiano, caen en una orientación: "religiosa, como una expresión de una religión post-bíblica y un anhelo por el reencantamiento del llamado mundo secular”. 
conocen sus procedimientos chamánicos es una forma de alejamiento y protección ante los peligros por el contacto con espíritus. Debido a este tipo de reflexiones etnográficas es que recientemente han surgido apuestas críticas para realizar etnografías de la separación, que no postulen la existencia a priori de relaciones ni de entidades como medios comprensivos (Candea, Cook, Trundle y Yarrow, 2015).

\section{Nuevas potencialidades}

Ante las críticas previamente expuestas, es necesario realizar dos precisiones. Primera, esta revisión no busca que se abandonen los autores abordados, sino que los lectores exploren con precaución sus obras, comprobando o confrontando las críticas expuestas. Segunda, las críticas descritas no deben ser generalizadas a los autores de cada ramificación, puesto que los teóricos caracterizados son parte de la primera generación de estos debates, y las siguientes generaciones poseen una postura crítica ante estos. Por eso proponen respuestas diferentes a sus problematizaciones comunes.

Ahora bien, las falencias de las teorías expuestas no agotan sus valiosos aportes para la disciplina. Desde la distancia crítica es necesario darles el mérito a Viveiros, Descola, Latour e Ingold por ofrecer nuevos interrogantes y modelos metodológicos experimentales para comprender y representar la realidad. Sus potencialidades y desaciertos le dejan grandes aprendizajes a la antropología. La alteridad invita a pensar comparativamente en la especificidad de los mundos y tiene como potencial su vigilancia reflexiva ante la extrapolación de categorías. La materialidad amplía la sensibilidad investigativa al conceptualizar las redes de agencia de no-humanos en articulación con las prácticas materiales que integran a los seres humanos. De este modo, los artefactos no son simplemente la expresión pasiva de la intencionalidad humana, sino los mediadores que limitan, potencian y configuran nuestras posibilidades de existencia. El énfasis del habitar lleva a ampliar la idea de la significación y la intencionalidad como cualidades exclusivamente humanas, puesto que construyen un robusto debate conceptual para señalar que somos parte de un mundo multiespecies en el que hacemos arreglos temporales con otras entidades para configurar los paisajes. Desde las rutas abiertas por los teóricos señalados, las nuevas generaciones de antropólogos están llamadas a construir nuevas apuestas investigativas por medio de la imaginación, la sensibilidad, la curiosidad y la crítica.

Precisamente las investigaciones antropológicas más potentes surgen en autores que se encuentran en un punto de yuxtaposición entre las diferentes ramificaciones caracterizadas. Piénsese, por ejemplo, en el trabajo de Kohn (2013), que, articulando los debates de la alteridad y el habitar, busca ampliar el estudio de la capacidad de 
representación a entidades no-humanas. Otro caso es la apuesta de Hornborg (2015), que retomando la vocación de la alteridad y los estudios materiales, analiza los objetos como sostén de mundos; de allí que la tecnología sea una forma de magia fetichista. A su vez, la investigación de Tsing (2015), cruzando el énfasis de la materialidad y del habitar, estudia la relación entre las cadenas capitalistas y los mundos orgánicos de los hongos matsutake.

Inclusive algunas investigaciones logran de forma paradigmática articular parcialmente las tres ramificaciones contemporáneas, como en el caso de Weismantel (2015) en su estudio de Chavín de Huantar. Por un lado, desde el habitar, dicho trabajo parte del interés por pensar la relación humanos-animales por los cuerpos multiespecies de las figuras talladas en piedras y las prácticas de caza de los chavines. Luego, por medio de la alteridad, retoma el perspectivismo de forma crítica para indagar en la multiplicidad de puntos de vista que componen estos tallados. Pero, a su vez, desde la materialidad, ve los tallados no como una hoja en blanco esperando la significación humana, sino como mediadores directos de la experiencia, puesto que "las piedras no simplemente representaban relaciones animales/humanas; ellas enactuaban una ontología animista del ver y conocer a través de las especies” (p. 147).

\section{Conclusiones}

El presente texto realizó una revisión de los debates ontológicos de la antropología. Este ejercicio no agotó su multiplicidad actual, sino que más bien mostró a grandes trazos las siluetas que componen las tendencias investigativas contemporáneas. La finalidad de dicha tarea fue presentarle al lector un amplio contexto analítico a partir del cual pueda desenvolver su estudio de forma más detallada y vigilante.

Estos debates, como se señaló, no son una ruptura del panorama antropológico, sino que retoman las preguntas comprensivas y representacionales de esta disciplina. Este camino académico fue posible porque su investigación comparativa, junto con la crisis del culturalismo, el biologicismo y la ciencia, condujo a que la antropología confrontara la generalización de sus antiguos pilares analíticos: naturaleza, cultura y sociedad.

Se indicó, además, que la categoría giro ontológico posee un uso demasiado heterogéneo que limita su especificidad analítica. Por este motivo, se trazó una ramificación de los debates ontológicos, identificando los énfasis de la alteridad, la materialidad y el habitar. Dicha operación estuvo acompañada de una caracterización 
evaluativa de sus autores más reconocidos. Este proceso tenía la finalidad de que, por un lado, el lector no esencializara las teorías antropológicas al poder ver cada debate individual como parte de un panorama diferenciado mucho más amplio; $y$, por el otro, que se identificara cómo dichos panoramas responden a problematizaciones legítimas y constitutivas de esta disciplina.

En síntesis, este texto buscó elaborar un diálogo problematizador para reconocer la complejidad de los interrogantes de los debates ontológicos y, en consecuencia, visibilizar los posibles logros y desaciertos de su experimentación contemporánea. Un aprendizaje central de este estudio es que los debates abordados no ofrecen nuevas premisas sobre la realidad, sino interrogantes abiertos que interpelan y permiten desarrollar nuevos problemas de investigación. Solo con el paso del tiempo se sabrá si estos debates poseen la fortaleza analítica para reinventarse productivamente, o si, por el contrario, su potencial se va a desvanecer en una empresa intelectual fallida. Por el momento, las preguntas de si es posible o no superar la división cultura/naturaleza, y representar la experiencia de mundo de los seres humanas junto con entidades no-humanas, seguirán abiertas.

Por último, en términos generales, el recorrido por los cambios históricos de la antropología indicó que esta no es una disciplina con un objeto, epistemología o metodología delimitada. Por ello los debates abordados parecieran no tener familiaridad con textos de Edward Tylor, puesto que esta disciplina no opera como un campo de consenso, sino más bien como una actividad práctica que delimita y problematiza lo humano por medio de múltiples objetos de estudio. Estas ideas implican que la antropología solo puede poseer una identidad parcialmente común en la medida en que reinvente sus pilares conceptuales. La mutación de la antropología, en este caso, bajo los debates ontológicos, paradójicamente es su misma condición de mantenimiento, puesto que su renovación le da consistencia y claridad a un esqueleto común de interrogantes sobre nuestra posición y relación con un mundo que buscamos conocer mientras nos descubrimos inmersos y descentrados de él.

\section{Sobre el autor}

Juan Camilo Perdomo-Marín. Antropólogo egresado de la Universidad de Caldas y docente de la misma institución. Sus estudios se enfocan en el debate cultura/naturaleza y la metodología de la investigación científica. Artículos recientes: "Desafiando el contexto: apuntes a los debates contemporáneos sobre redes y escalas” (2020) y “Agencias, mundos y ontologías como escenarios de problematización de la antropología contemporánea” (2020). 


\section{Referencias}

Abu-Lughod, L. (2012). Escribir contra la cultura. Andamios. Revista de Investigación Social, 9(19), 129-157.

Appadurai, A. (1991). Introducción: Las mercancías y las políticas del valor. La vida social de las cosas. Perspectiva cultural de las mercancías (pp. 17-87). México: Grijalbo.

Arhem, K. (2001). La red cósmica de la alimentación. La interconexión de humanos y naturaleza en el noreste de la amazonia. En P. Descola y G. Pálsson (Coord.), Naturaleza y sociedad: perspectivas antropológicas (pp. 214-236). México: Siglo XXI Editores.

Bird-David, N. (1999). "Animism” revisited: personhood, environment, and relational epistemology. Current Anthropology, 40(1), 67-91.

Blaser, M. (2009). Ontología política un programa de caza sustentable. Red de Antropologías del Mundo, 4, 82-109. Recuperado de: http://ram-wan.net/old/documents/05_e_Journal/journal-4/jwan4.pdf

Blaser, M. y De la Cadena, M. (2009). Introducción. Red de Antropologías del Mundo, 4, 3-9. Recuperado de http://ram-wan.net/old/documents/05_e_Journal/journal-4/jwan4.pdf

Brabec de Mori, B. (2013). Shipibo laughing songs and the transformative faculty: performing or becoming the other. Ethnomusicology Forum, 22(3), 343-361.

Bryant, L., Srnicek, N. y Harman, G. (2011). Towards a speculative philosophy. En L. Bryant, N. Srnicek y G. Harman (Eds.), The speculative turn: continental materialism and realism (pp.1-18). Australia: Repress.

Callon, M. (1986). Some elements of a sociology of translation: domestication of the scallops and the fishermen of St Brieuc Bay. En J. Law (Ed.), Power, action and belief: a new sociology of knowledge? (pp. 196-223). Londres: Routledge.

Candea, M. (2012). Internal others ethnographies of naturalism. Cambridge Anthropology, $30(2), 36-47$.

Candea, M., Cook, J., Trundle, C. y Yarrow, T. (2015). Introduction: reconsidering detachment. M. Candea, J. Cook, C. Trundle y T. Yarrow (Eds.), Detachment: essays on the limits of relational thinking (pp. 1-31). Manchester: Manchester University Press.

Castellanos, D. (2019). Vasijas envidiosas de Aguabuena: un ensayo etnográfico sobre la vida del mundo material. En L. A. Suárez-Guava (Ed.), Cosas vivas: antropología de objetos, sustancias y potencias (pp. 51-70). Bogotá: Editorial Pontificia Universidad Javeriana.

De la Cadena, M. (2010). Indigenous cosmopolitics in the Andes: conceptual reflections beyond "politics". Cultural Anthropology, 25(2), 334-370.

Descola, P. y Pálsson, G. (Coords.) (2001). Naturaleza y sociedad: perspectivas antropológicas . México: Siglo XXI Editores. 
Descola, P. (2001). Construyendo naturalezas: ecología simbólica y práctica social. En P. Descola y G. Pálsson (Coords.), Naturaleza y sociedad: perspectivas antropológicas (pp. 101123). México: Siglo XXI Editores.

Descola, P. (2012). Más allá de la naturaleza y la cultura. Buenos Aires: Amorrortu Editores.

Descola, P. (2014). All too human (still) a comment on Eduardo Kohn's How forests think. Hau: Journal of Ethnographic Theory, 4(2), 267-273.

Descola, P. (2016). Biolatry: a surrender of understanding (response to Ingold's A Naturalist Abroad in the Museum of Ontology). Anthropological Forum, 26(3), 321-328.

Escobar, A. (2010). Territorios de la diferencia: lugar, movimientos, vidas, redes. Popayán: Envión Editores.

Golub, A. (2014). Ontology and wonder: an interview with Michael W. Scott. Recuperado de https://savageminds.org/2014/03/19/ontology-and-wonder-an-interview-with-michael-w-scott/

Graeber, D. (2015). Radical alterity is just another way of saying "reality". A reply to Eduardo Viveiros de Castro. Hau:Journal of Ethnographic Theory, 5(2), 1-41.

Gupta, A. y Ferguson, J. (2008). Más allá de la “cultura”: espacio, identidad y las políticas de la diferencia. Antípoda, 7, 233-256.

Halbmayer, E. (2012). Amerindian mereology: Animism, analogy, and the multiverse. India$n a, 29,103-125$.

Hanks, W. y Severi, C. (2014). Translating worlds: the epistemological space of translation. Hau: Journal of Ethnographic Theory, 4(2), 1-16.

Hastrup, K. (2013). Scales of attention in fieldwork: global connections and local concerns in the Arctic. Ethnography, 14(2), 145-164.

Hastrup, K. (Ed.). 2014. Anthorpology and nature. Nueva York: Routledge.

Helmreich, S. (2009). Alien ocean: anthropological voyages in microbial seas. Los Ángeles: University of California Press.

Henare, A., Holbraad, M. y Wastell, S. (Eds.) (2007). Thinking through things: theorising artefacts ethnographically. Nueva York: Routledge.

Hicks, D. (2010). The material-cultural turn: event and effect. D. Hicks y M. C. Beaudry (Eds.), The Oxford Handbook of material culture studies (pp. 25-98). Oxford: Oxford University Press.

Holbraad, M. y Pedersen, M. (2017). The ontological turn: an anthropological exposition. Reino Unido: Cambridge University Press.

Holbraad, M. (2009). Ontology, ethnography, archaeology: an afterword on the ontography of things. Cambridge Archaeological Journal, 19(3), 431-441.

Hornborg, A. (2015). The political economy of technofetishism. Agency, Amazonian ontologies, and global magic. Hau:Journal of Ethnographic Theory, 5(1), 35-57. 
Hornborg, A. (2017). Artifacts have consequences, not agency: toward a critical theory of global environmental history. European Journal of Social Theory, 20(1), 95-110.

Hugh-Jones, S. (2019). Rhetorical antinomies and radical othering. Recent reflections on responses to an old paper concerning human-animal relations in Amazonia. Hau:Journal of Ethnographic Theory, 9(1), 162-171.

Hvenegaard, H. (2016). Chaosmology: shamanism and personhood among the Bugkalot. Hau: Journal of Ethnographic Theory, 6(1), 189-205.

Ingold, T. (1993). The art of translation in a continuous world. En G. Palsson (Ed.), Beyond boundaries: understanding, translation, and anthropological discourse (pp. 210-230). Oxford: Berg.

Ingold, T. (1994). The animal in the study of humanity. En T. Ingold (Ed.), What is an animal? (pp. 84-99). Gran Bretaña: Routledge.

Ingold, T. (2000). The perception of the environment: essays on livelihood, dwelling and skill. Londres: Routledge.

Ingold, T. (2011). Being alive: essays on movement, knowledge and description. Nueva York: Routledge.

Ingold, T. (2013a). Consideraciones de un antropólogo sobre la biología. En L. Montenegro (Ed.), Cultura y naturaleza: aproximaciones a propósito del bicentenario de la independencia de Colombia (pp. 100-132). Bogotá: Jardín Botánico José Celestino Mutis.

Ingold, T. (2013b). Anthropology beyond humanity. Suomen Antropologi: Journal of the Finnish Anthropological Society, 38(3), 5-23.

Ingold, T. (2015a). Líneas: una breve historia. Barcelona: Gedisa.

Ingold, T. (2015b). The life of lines. Nueva York: Routledge.

Ingold, T. (2016a). "A naturalist abroad in the Museum of Ontology: Philippe Descola's beyond nature and culture". Anthropological Forum, 26(3), 301-320.

Ingold, T. (2016b). Rejoinder to Descola's Biolatry: a surrender of understanding. Anthropological Forum, 26(3), 329-332.

Jaramillo, P. (2020). Mining leftovers: making futures on the margins of capitalism. Cultural Anthropology, 35(1), 48-73.

Keane, W. (2018). A minimalist ontology, with other people in it. Hau:Journal of Ethnographic Theory, 8, 45-47.

Kirksey, E. y Helmreich, S. (2010). The emergence of multispecies ethnography. Cultural Anthropology, 25(4), 545-576.

Kohn, E. (2013). How forests think. Toward an anthropology beyond the human. Los Ángeles: University of California Press.

Latour, B. (2007). Nunca fuimos modernos: ensayos de antropología simétrica. Buenos Aires: Siglo XXI Editores. 
Latour, B. (2008). Reensamblar lo social: una introducción a la teoría del actor-red. Buenos Aires: Ediciones Manantial.

Law, J. (2004). After method: mess in social science research. Estados Unidos: Routledge.

Londoño Sulkin, C. (2005). Inhuman beings: morality and perspectivism among Muinane People (Colombian Amazon). Ethnos, 70(1), 7-30.

Micarelli, G. (2018). Soberanía alimentaria y otras soberanías: el valor de los bienes comunes. Revista Colombiana de Antropología, 54(2), 119-142.

Milton, K. (1996). Environmentalism and cultural theory. Exploring the role of anthropology in environmental discourse. Londres: Routledge.

Mol, A. (2002). Body multiple. Ontology in medical practice. Estados Unidos: Duke University Press

Morita, A. (2013). The ethnographic machine: experimenting with context and comparison in Strathernian Ethnography. Science, Technology, \& Human Values, 39(2), 214-235.

Povinelli, E. (1995). A do rocks listen? The cultural politics of apprehending Australian aboriginal labor. American Anthropologist, 97(3), 505-518.

Pederson, M. (2013). The fetish of connectivity. En G. Evans, E. Silva y N. Thoburn (Eds.), Objects and materials: a routledge companion (pp. 197-207). Londres: Routledge.

Ramos, A. (2012). The politics of perspectivism. Annual Reviews Anthropology, 41, 481-494.

Ress, T. (2016). On deanthropologizing anthropology - An essay on Tarek Elhaik's "The incurable image". Recuperado de http://somatosphere.net/2016/12/on-deanthropologizinganthropology-an-essay-on-tarek-elhaiks-the-incurable-image.html

Rocheleau, D. (2011). Rooted networks, webs of relation, and the power of situated science: bringing the models back down to earth in Zambran. En M. J. Goldman, P. Nadasdy y M. D. Turner (Eds.), Knowing nature: conversations at the intersection of political ecology and science studies (pp. 209-226). Chicago: University of Chicago Press.

Ruiz-Serna, D. y Del Cairo, C. (2016). Los debates del giro ontológico en torno al naturalismo moderno. Revista de Estudios Sociales, 55, 193-204.

Ruiz-Serna, D. (2017). El territorio como víctima. Ontología política y las leyes de víctimas para comunidades indígenas y negras en Colombia. Revista Colombiana de Antropología, 53(2), 85-113.

Sahlins, M. (1988). Cultura y razón práctica. Barcelona: Gedisa.

Sahlins, M. (2014). On the ontological scheme of beyond nature and culture. Hau: Journal of Ethnographic Theory, 4(1), 281-290.

Santos-Granero, F. (2012). Introducción. Perspectivas construccionales del mundo en la Amazonía indígena. En F. Santos-Granero (Ed.), La vida oculta de las cosas. Teorías indígenas de la materialidad y la personeidad (pp. 13-51). Quito: Abya Yala.

Sapir, E. (1963). The status of linguistics as a science. Selective writings of Edward Sapir (pp. 160-178). California: University of California. 
Scott, M. (2016). To be Makiran is to see like Mr. Parrot: the anthropology of wonder in Solomon Islands. Journal of the Royal Anthropological Institute, 22(3), 1-22.

Simonetti, C. y Espirito, D. (2016). Entrevista a Tim Ingold. Chungara, 48(4), 487-502.

Strathern, M. (1980). No nature, no culture: the Hagen case. En C. MacCormaek y M. Strathern (Eds.), Nature, culture and gender (pp. 174-222). Cambridge: Cambridge University Press.

Strathern, M. (1996). Cutting the network. The Journal of the Royal Anthropological Institute, 2(3), 517-535.

Strathern, M. (2004). Partial connections. Altamira Press: Walnut Creek.

Strathern, M., Peel, J., Toren, C. y Spencer, J. (1996). The concept of society is theoretically obsolete. En T. Ingold (Ed.), Key debates in anthropology (pp. 55-98). Londres: Routledge.

Suárez-Guava, L. (2019). La vida de las cosas y las formas del conocimiento: desafíos para hacer otras antropologías. En L. A. Suárez-Guava (Ed.), Cosas vivas. Antropología de objetos, sustancias y potencias (pp. 19-48). Bogotá: Editorial Pontificia Universidad Javeriana.

Swanson, H., Bubandt, N. y Tsing, A. (2015). Less than one but more than many. Anthropocene as science fiction and scholarship-in-the-making. Environment and Society: Advances in Research, 6, 149-166.

Tsing, A. (2010). Worlding the matsutake diaspora: or, can actor-Network theory experiment with holism? En T. Otto y N. Bubandt (Eds.), Experiments in Holism: Theory and Practice in Contemporary Anthropology (pp. 47-66). Reino Unido: Blackwell.

Tsing, A. (2015). The mushroom at the end of the world. On the possibility of life in capitalist ruins. Reino Unido: University Press.

Venkatesan, S., Martin, K., Scott, M., Pinney, C., Ssorin-Chaikov, N., Cook, J. y Strathern, M. (2013). The group for debates in anthropological theory (GDAT), The University of Manchester: the 2011 annual debate-non-dualism is philosophy not ethnography. Critique of Anthropology, 33(3), 300-360.

Viveiros de Castro, E. (2004). Perspectival anthropology and the method of controlled equivocation. Tipiti:Journal of the Society for the Anthropology of Lowland South America, 2(1), 3-22.

Viveiros de Castro, E. (2010). Metafísicas caníbales: líneas de antropología postestructural. Buenos Aires: Katz Editores.

Viveiros de Castro, E. (2015). Who is Afraid of the Ontological Wolf? Some Comments on an Ongoing Anthropological. The Cambridge Journal of Anthropology, 33(1), 2-17.

Vilaça, A. (2015). Do animists become naturalists when converting to Christianity? Discussing an ontological turn. The Cambridge Journal of Anthropology, 33(2), 3-19.

Wagner, R. (1981). The assumption of culture. The invention of culture (pp. 1-16). Chicago: University of Chicago Press.

Weismantel, M. (2015). Seeing like an archaeologist: Viveiros de Castro at Chavín de Huantar. Journal of Social Archaeology, 15(2), 139-159.

Wolf, E. (1988). Inventing society. American Ethnologist, 15(4), 752-761. 